\title{
AVALIAÇÃO DA EFICIÊNCIA NA ALOCAÇÃO DOS RECURSOS ECONÔMICOS FINANCEIROS NO ÂMBITO HOSPITALAR
}

\section{EVALUATION OF EFFICIENCY IN THE ALLOCATION OF RESOURCES IN HOSPITAL FINANCIAL ECONOMIC CONTEXT}

\section{EVALUACIÓN DE LA EFICIENCIA EN LA ASIGNACIÓN DE RECURSOS EN CONTEXTO ECONÓMICO FINANCIERA DEL HOSPITAL}

\author{
Patrícia Valeriano Trivelato \\ Univiçosa \\ patricia.trivelatto@gmail.com
}

\author{
Michelle Barbosa Soares \\ Universidade Federal de Viçosa \\ msmichellesoares@yahoo.com.br
}

\section{Wagner Gonçalves Rocha \\ Universidade Federal de Viçosa \\ wagner_meleta@yahoo.com.br}

\section{Evandro Rodrigues de Faria}

Universidade Federal de Viçosa

evandro_farias@yahoo.com.br
Submetido em: 22/12/2014

Correções obrigatórias: 13/02/2015

Artigo aceito em: 07/09/2015

\section{Resumo}

Esta pesquisa desenvolve um exercício de avaliação alocativa da eficiência de 35 hospitais, dentre os quais estão hospitais públicos, filantrópicos e privados. O objetivo principal é avaliar se existe diferença na alocação de recursos econômico-financeiros entre esses tipos de hospitais. A metodologia é a análise envoltória de dados (Data Envelopment Analysis - DEA), em combinação com testes e procedimentos estatísticos não paramétricos. A pesquisa também explora extensões, como as orientações que o DEA fornece para ajustes nas eficiências observadas, relativas ao problema de retorno de escala, e associações dos índices financeiros com formação de interação entre a eficiência e os indicadores financeiros. Ao longo do texto, procura-se oferecer elementos aos leitores para apreciar o modelo DEA como um mecanismo de avaliação comparativa dos diferentes hospitais. De forma geral, apesar das dificuldades inerentes, o estudo presente e os demais têm demonstrado que a ausência de informações confiáveis com relação à eficiência do custo hospitalar é um dos principais problemas dos hospitais, o que dificulta os esforços para melhorar a eficiência na prestação de serviço.

Palavras-Chave: Eficiências; Hospitais, Alocação de recursos.

\begin{abstract}
This research is an exercise in assessing of the allocative efficiency of hospitals 35 of them are public, philanthropic and private hospitals. The main objective is to assess whether there are differences in the allocation of financial and economic resources between types of hospitals. The methodology is Data Envelopment Analysis (DEA) in combination with non - parametric statistical tests and procedures. The research also explores extensions, such as the guidelines that DEA provides for adjustments to the observed efficiencies for the recurring problem of scale, and associations of financial ratios with formation of interaction between efficiency and financial indicators. Throughout the text, if you want to offer elements for readers to appreciate the DEA model as a mechanism for benchmarking different hospitals. Overall, despite the difficulties inherent in this study and others have shown that the absence of reliable information on the efficiency of hospital costs is a major problem in hospitals, which complicates efforts to improve efficiency in service delivery.
\end{abstract}

Keywords: Efficiencies; Hospitals, Resource Allocation. 


\section{Resumen}

Esta investigación desarrolla una evaluación del ejercicio de asignación de 35 hospitales de las cuales son públicas, filantrópica y hospitales privados. El objetivo principal es evaluar si existen diferencias en la asignación de los recursos financieros y económicos entre los tipos de hospitales. La metodología es el análisis envolvente de datos (DEA Análisis Envolvente de Datos) en combinación con las pruebas y procedimientos estadísticos no paramétricos. La investigación también explora las extensiones, como las directrices que la DEA prevé ajustes a las eficiencias observadas para el problema recurrente de escala, y las asociaciones de relaciones financieras con la formación de la interacción entre la eficiencia y los indicadores financieros. A lo largo del texto, elementos que buscan ofrecer a los lectores a apreciar el modelo DEA como un mecanismo de evaluación comparativa de los diferentes hospitales. En general, a pesar de las dificultades inherentes a este estudio y otros han demostrado que la falta de información fiable sobre de la eficiencia de los costos hospitalarios es un gran problema en los hospitales, lo que complica los esfuerzos para mejorar la eficiencia en la prestación de servicios.

Palabras clave: Eficiencias; Hospitales, asignación de recursos.

\section{INTRODUÇÃO}

Segundo Souza (2012), as entidades hospitalares nos últimos anos não têm apresentado bons resultados com relação à gestão dos recursos econômico-financeiros. Partindo do pressuposto de que os hospitais são responsáveis pela promoção à saúde, por serem a primeira opção quanto à busca de atendimento médico, e considerando à insuficiência dos recursos existentes, esse panorama se torna motivo de grande preocupação.

Os valores destinados à saúde são altamente expressivos em todo o mundo. No Brasil os Hospitais são o centro do sistema de saúde, sendo responsáveis por dois terços dos gastos do setor e parte dos serviços produzidos. Boa parte dos hospitais dependem de financiamento público, pois financiam prestadores de serviços privados com os recursos públicos (LA FORGIA; COUTTOLENC, 2009).

Uma das causas possíveis pode ser a falta de financiamento por parte do poder público. Dados recentes apontam que, enquanto em outros países o repasse destinado à saúde chega a $20 \%$ do Produto Interno Bruto (PIB), o Brasil destina apenas cerca $8 \%$ dos seus recursos, ficando abaixo da média mundial de 11,7\% (OMS, 2011).

Em contrapartida, o gasto com saúde tem aumentado rapidamente nos últimos anos, sendo primordial que seja otimizada a alocação dos recursos econômico-financeiros com eficiência. $\mathrm{O}$ crescimento tecnológico, em especial nas últimas cinco décadas, gerou um aumento de gastos em saúde em todo o mundo (CALVO, 2002; ROTTA, 2004).

Contudo, para avaliar, de maneira mais aprofundada, se os recursos destinados à saúde são suficientes e geridos com eficiência, deve-se focar na rede hospitalar por se tratarem dos órgãos mais relevantes na promoção da saúde à população. No Brasil, segundo pesquisas, cerca de $70 \%$ dos recursos da saúde são em atenção aos hospitais, sendo uma estimativa anual (LA FORGIA; COUTTOLENC, 2009).

Pode-se creditar tais fatos à alta complexidade em administrar os recursos captados e à variedade de serviços existentes em uma organização hospitalar, todos esses fatores aliados ao crescimento frenético da tecnologia.

Tendo em vista que os serviços de saúde devem ser eficientes microeconomicamente (controlando os custos inerentes) e macroeconomicamente (maximizando os serviços prestados e minimizando os custos), é importante que se tenha como premissas as diretrizes para a utilização dos recursos, maximizando o resultado com recursos fixos (CESCONETTO et al., 2008). Assim, a ausência de informações acerca da eficiência e dos custos da atenção hospitalar é um dos principais problemas das entidades hospitalares, o que prejudica os planejamentos e as ações de melhoria na eficácia (RAIMUNDINI et al., 2004). 
Dentro do contexto, La Forgia e Couttolenc (2009) compartilham do mesmo pensamento, ressaltando que é cada vez mais urgente conter os gastos e com isso poder aplicar melhor os recursos existentes. É fundamental, nos dias atuais, que as organizações hospitalares sejam administradas de forma eficiente, focando em bons resultados. Os hospitais prestadores de serviços privados apresentam uma grande oportunidade de maximização do lucro; já nos hospitais públicos e filantrópicos, tem-se a maximização dos orçamentos.

Dessa forma, faz-se necessário avaliar o grau de eficiência dos hospitais filantrópicos, públicos e privados, principalmente no que se refere à alocação dos recursos existentes. Sendo assim, o trabalho propõe discutir se os recursos econômico-financeiros nos hospitais filantrópicos, públicos e privados estão sendo alocados de forma eficiente e eficaz, o que conduz ao seguinte problema de pesquisa: Existe diferença na eficiência da gestão econômicofinanceira entre os hospitais filantrópicos, públicos e privados?

\section{A SAÚDE PÚBLICA BRASILEIRA}

O setor de saúde no Brasil faz parte de um amplo e complexo sistema, condicionado por variáveis de políticas sociais e econômicas, em que se determina o grau de eficiência no planejamento e administração do setor, influenciando diretamente no risco econômicofinanceiro das entidades hospitalares (MARRACINI, 2002).

O cenário da saúde pública no Brasil sempre foi grande motivo de preocupação. Embora os primórdios da Saúde Pública tenham tido início em 1808, só em 1953, com a Lei nº 1.920, o Ministério da Saúde veio a ser constituído, passando por diversas reformas.

A descentralização foi, sem dúvida, a característica definidora da reforma sanitária para a criação do Sistema Único de Saúde (SUS). O movimento sanitário tinha como discussão os efeitos maléficos do sistema implantado sobre a saúde da população. A característica principal era a construção de um novo modelo, o Sistema Único de Saúde, o qual deveria substituir os sistemas de saúde até então adotados pelo governo. O SUS tem como característica a integralidade do atendimento, a universalização, descentralização de poder, a eficácia e eficiência na qualidade do serviço prestado, sendo democrático no sentido de garantir a participação da população através do Conselho de Saúde. (FONSECA; FERREIRA, 2009).

Foi em meados dos anos 1980 que o Brasil passou pela reforma mais ambiciosa, em que se mudou a estrutura, a organização e o financiamento na área da saúde, o que resultou na criação do Sistema Único de Saúde (SUS), resultado de quase uma década de trabalho. A Constituição definiu o SUS como o principal prestador de serviços à saúde no Brasil, ressaltando que o acesso aos serviços prestados pelo SUS é universal e gratuito (LA FORGIA; COUTTOLENC, 2009).

Apesar de o SUS ter sido regulamentado em 1990, isso por várias questões políticas e econômicas, devido ao carácter de privatização do Estado, diante do citado supra o sistema ainda não conseguiu ser implementado na sua magnitude, porém apresenta diretrizes que são o caminho para a melhoria do sistema de saúde no Brasil. (FONSECA; FERREIRA, 2009).

Contudo, as problemáticas que envolvem o setor de saúde no Brasil são muitas, destacando-se a existência de uma crise que vem da própria estrutura dos serviços de saúde no país, onde a política de saúde aponta dois caminhos: a privatização dos serviços, em que a maior parte da população estaria excluída do acesso à saúde; e a luta incessável pela manutenção do direito conquistado na Constituição Federal de 1988, a qual garante o acesso universal e igualitário ao serviço de qualidade. (FONSECA; FERREIRA, 2009).

O sistema brasileiro de saúde, segundo Fonseca e Ferreira (2009), baseia-se em um modelo voltado à doença já manifestada, ou seja, na maioria das vezes o paciente está em um estágio avançado. Portanto, por ser um sistema direcionado aos hospitais, torna-se muito custosa a saúde no país. 
Embora sejam o componente predominante do sistema de saúde no Brasil, os hospitais até recentemente não vinham recebendo atenção tanto por parte dos formuladores de políticas como dos pesquisadores. (LA FORGIA; COUTTOLENC, 2009).

Os hospitais estão entre as mais complexas organizações a serem administradas, pois em um hospital encontram-se reunidos vários serviços e situações distintas e simultâneas, podendo ser um hotel, lavanderia, oferecer serviços de limpeza, vigilância, médicos, restaurantes, tudo isso para atender ao usuário. (CELESTINO, 2002).

Wolff (2005) compartilha do mesmo pensamento: os hospitais caracterizam-se como organizações complexas e realizam atividades complexas, principalmente.

O setor hospitalar brasileiro é amplo, com cerca de 6.690 unidades e com aproximadamente 463.166 mil leitos, compreendendo uma multiplicidade de arranjos de financiamentos, tipos de propriedade e arranjos organizacionais (LOBO, 2010).

Calvo (2002) salienta que os hospitais são considerados, no setor da saúde, as entidades com maior potencial de lucro. No Brasil, como no mundo, os hospitais apresentam muitas diferenças entre si, porém o que mais difere um hospital do outro, do ponto de vista econômico, é a sua gestão e propriedade, dadas às condições jurídicas e de financiamento do sistema de saúde.

Contudo, Raimundini (2003) ressalta que os hospitais têm enfrentado grandes dificuldades em definir os preços dos serviços prestados, uma vez que não possuem controle adequado dos custos incorridos e dos recursos consumidos na prestação desses. Além da determinação dos custos, outro fator determinante na gestão financeira é a especialidade dos procedimentos realizados.

Em razão de restrições orçamentárias, é de interesse de toda sociedade e dever dos gestores, respeitados os parâmetros técnicos, os recursos disponíveis e as especialidades hospitalares, que o sistema opere com máxima eficiência, transformando insumos em produtos e serviços. Assim, a necessidade de se obter melhor aproveitamento dos recursos disponíveis torna-se cada vez mais primordial (COSTA; CASTANHAR, 2003).

Para Marinho e França (2001), a mensuração da eficiência no setor saúde em países pobres, como o Brasil, é fundamental. Contudo, não parece justo e, tão pouco, aceitável atender à crescente demanda social por serviço de saúde através, tão somente, do financiamento por impostos, sem repensar as formas como os recursos têm sido explorados.

Bittar (2001) ressalta que a estrutura hospitalar possui quatro grandes componentes: recursos humanos, recursos materiais permanentes, recursos de consumo e recursos de utilidade pública. Tais componentes se distribuem em áreas e subáreas diferenciadas, que apresentam características específicas. Essa complexidade das organizações, que incluem os hospitais filantrópicos, públicos e privados, necessita uma gestão eficiente que viabilize os bons resultados econômico-financeiros. seguir.

Os hospitais são compostos por três subsetores principais, sobre os quais tratamos a

\section{HOSPITAIS FILANTRÓPICOS}

Os hospitais filantrópicos são caracterizados pelo fato de serem conveniados ao SUS, sendo necessária a destinação de no mínimo $60 \%$ de ocupação e prestação de serviços ao SUS, obtendo assim recursos financeiros e isenções tributárias. O setor filantrópico precisa ser considerado de acordo com sua diversidade, exigindo, portanto, diferentes tratamentos e ações, como afirmam Portela et al (2004).

Segundo o CNE - Cadastro Nacional de Estabelecimento de Saúde, o Brasil conta com 1.911 hospitais filantrópicos. 


\section{HOSPITAIS PÚBLICOS}

Quanto aos hospitais públicos, sua principal característica é o convênio com o Sistema Único de Saúde. A maior parte de seus recursos é proveniente do orçamento público, o qual depende diretamente de arrecadação. Os hospitais públicos integram o patrimônio da União, do Estado, dos Municípios e do Distrito Federal, ou seja, equivalem a pessoas jurídicas de direito público (BRASIL, 1975). Na esfera pública, o Brasil conta com 2.142 hospitais.

O gestor do hospital público segue orientações da política pública de saúde estabelecida pelo Conselho de Saúde, o qual é formado por representantes de três setores - os usuários, os trabalhadores e os administradores, que buscam priorizar seus objetivos de atuação os serviços de saúde, harmonizando os conflitos sociais (CALVO, 2002).

\section{HOSPITAIS PRIVADOS}

Os hospitais de direito privado integram o patrimônio de uma pessoa, seja ela física ou jurídica de direito privado, que não seja instituída pelo poder público (BRASIL, 1975). Segundo dados da Federação Brasileira de Hospitais, tendo como fonte principal o CNES, o total de hospitais privados no Brasil em abril de 2012 era de 2.637, hospitais esses com finalidade de lucratividade.

Braga e Goes de Paula (1981) afirmam que, no Brasil, o setor privado tem desempenhado um papel relevante na prestação de serviços na área da saúde nas últimas décadas. As quais são financiadas diretamente com os recursos públicos.

\section{EFICIÊNCIA NA GESTÃO DOS HOSPITAIS}

Na economia, quando se trata de eficiência, refere-se à otimização de recursos e à ausência de desperdício. Contudo, a eficiência se dá pela utilização máxima dos recursos existentes para satisfazer as necessidades e os desejos, seja de um indivíduo ou organizações (PINDYCK; RUNBINFELD, 1994). Em corroboração, Fonseca e Ferreira (2009) salientam que a eficiência é entendida, sob uma forma geral, como a capacidade, ou até mesmo habilidade, de fazer o uso mais adequado dos recursos que se tem à disposição, a fim de alcançar o resultado pretendido.

Segundo Gomes e Baptista (2004), a eficiência é segregada em dois componentes: a eficiência técnica, que reflete a habilidade de obter máximo produto, dado um conjunto de insumos; e a eficiência alocativa, que é a habilidade em utilizar os insumos em proporções ótimas, dados seus preços relativos. Essas medidas constituem a medida de eficiência econômica total.

Miller (1981) caracteriza a eficiência como a relação entre produto e insumo, sendo medida pelas unidades físicas do produto comparadas às unidades físicas dos insumos. Assim, a eficiência é uma medida de desempenho relativa, sendo que a melhor prática é tida como parâmetro para análise dos demais casos.

Para Souza et al. (2008), para atingir o objetivo de prestação de serviços de saúde à população, é importante que os gestores hospitalares realizem avaliações a fim de mensurar a eficiência da gestão. 
Contudo, no Brasil são poucas as pesquisas relacionadas ao grau de eficiência utilizando a DEA, chega a ser menor ainda na área da saúde com o foco em hospitais. Existem poucas pesquisas que estabelecem a diferença da eficiência na gestão de recursos econômicofinanceiros entre a linha filantrópica, publica e privada em âmbito nacional que empregam o método tanto para retornos constantes de escalas quanto para retornos variáveis. (LA FORGIA; COUTTOLENC, 2009).

No Brasil, o primeiro estudo que utilizou a Análise Envoltória de Dados para verificar a eficiência dos hospitais foi o de Marinho (2001), que analisou a eficiência de quatro hospitais públicos e dois privados localizados no município de São Paulo.

Por sua vez, Cesconetto et al. (2008) avaliam a eficiência de 112 hospitais gerais com mais de $80 \%$ conveniados ao SUS do estado de Santa Catarina. Os autores empregaram o modelo de DEA para retornos variáveis e verificaram, ao concluir a pesquisa, que a maioria dos hospitais eficientes são filantrópicos e de pequeno porte.

Em outro estudo, Lima et al. (2004) destacam que, quando se relaciona o nível de complexidade assistencial à capacidade operacional, quase todos os hospitais filantrópicos de baixa e média complexidade apresentam uma gestão ineficiente.

$\mathrm{Na}$ linha de pesquisa de hospitais, podemos citar os trabalhos de Lins et al (2007) e Gonçalves et al. (2007), que mensuram o desempenho de hospitais públicos e universitários no território brasileiro.

Sob a percepção da análise da eficiência, pretende-se identificar os hospitais eficientes e demonstrar os pontos para que os hospitais de baixa eficiência otimizem a alocação dos recursos econômico-financeiros (SEDIYAMA et al., 2012).

\section{METODOLOGIA}

Quanto aos objetivos da pesquisa, trata-se de uma pesquisa descritiva, pois procura-se mostrar, através da utilização da Análise Envoltória de Dados, os indicadores do grau de eficiência nos hospitais sob análise e expor, assim, diferenças de eficiência. Segundo Gil (2010), as pesquisas descritivas são aquelas que têm como objetivo a descrição das características de uma determinada população, ou podendo identificar possíveis reações entre as variáveis.

Quanto aos meios de investigação, será utilizada como principal instrumento na coleta de dados a pesquisa documental, que será realizada através de documentos, em sites oficiais que disponibilizem indicadores e resultados no âmbito hospitalar (CNES, DATASUS, Ministério da Saúde, Balanços Sociais) e que divulguem informações quanto aos gastos e desempenho dos hospitais brasileiros.

Serão utilizadas as informações disponíveis no Cadastro Nacional dos Estabelecimentos de Saúde Brasil, também pelo DATASUS, CNES que têm como fonte o Ministério da Saúde. A base de dados da pesquisa será a mais recente possível, considerando o ano de 2012 como referência. Dentre os diversos dados fornecidos por essas fontes, pode-se citar: internações hospitalares; produção ambulatorial; leitos em uso; funcionários; médicos cadastrados; e cirurgias realizadas.

\section{CARACTERIZAÇÃO DA AMOSTRA}

No Brasil existem atualmente 6.690 hospitais, distribuídos em $21 \%$ na esfera municipal, $8 \%$ estadual, $1 \%$ federal e $70 \%$ privados, segundo dados da Federação Brasileira de Hospitais (2012).

Dentre estes, serão escolhidos, aleatoriamente, 35 hospitais, distribuídos entre filantrópicos, públicos e privados, os quais irão compor uma amostra estratificada. 
O objetivo da amostra estratificada é garantir que cada estrutura hospitalar citada tenha um ou mais representantes com características semelhantes para que, futuramente à análise de eficiência, possam ser utilizadas como parâmetros entre os hospitais brasileiros para apontar se existe diferença de eficiência entre os hospitais filantrópicos, públicos e privados no território nacional.

\section{ANÁLISE E TRATAMENTO DOS DADOS}

Para alcançar o objetivo geral proposto, foi escolhida uma abordagem metodológica de natureza quantitativa. Ressalta Gil (2010) que, ao se referir ao cunho quantitativo da pesquisa, os resultados são organizados em tabelas e submetidos a testes estatísticos.

Quanto à mensuração da eficiência, a priori, será utilizada a abordagem Análise Envoltória de Dados (DEA), com utilização do modelo CCR, com orientação para o produto.

\section{MENSURAÇÃO DA EFICIÊNCIA POR INTERMÉDIO DA DEA}

O método DEA é uma técnica de Pesquisa Operacional (PO) que tem como base a Programação Linear convencional e fracionária, podendo ser aplicada sob diferentes maneiras. A PO é uma abordagem científica aplicada à tomada de decisões que envolvem as operações de sistemas organizacionais (ATHAYDE, 2003). Essa é uma técnica não paramétrica na programação linear, cujos primeiros estudos foram desenvolvidos por Charnes, Cooper e Rhodes (1978), baseados no trabalho de Farrel (1957).

A proposta inicial desenvolvida por Farrell (1957) sugere um modelo em que se comparava as eficiências das organizações com a melhor eficiência até então observada, ao invés de compará-la com algum ideal inatingível. No entanto, este modelo considerava um único input e um único output.

A partir disso, Charnes et al. (1978) ampliaram os seus estudos com base nos múltiplos inputs e outputs, por meio de modelos não paramétricos denominados de Data Envelopment Analysis (DEA) ou Análise Envoltória de Dados, com retorno constante de escala, denominado modelo CCR (homenagem aos autores) ou CRS (Constant Returns to Scale). O modelo CCR pressupõe retorno constante de escala, isto é, acréscimos no consumo de recursos resultam em aumento proporcional na quantidade obtida de produto. Este modelo permite uma avaliação objetiva da eficiência global e identifica as fontes e estimativas de montantes das ineficiências identificadas (CASA NOVA, 2002).

Banker et al. (1984), eliminando a necessidade de rendimentos constantes de escala, desenvolveram uma nova modelagem para o DEA, assumindo um retorno variável de escala, denominado BCC ou VRS (Variable Returns to Scale). Este modelo estabelece distinção entre ineficiências técnicas e de escala, estimando a eficiência técnica pura, a uma dada escala de operações, e identificando se estão presentes ganhos de escala crescente, decrescente e constante, para futura exploração (CASA NOVA, 2002).

Segundo Casa Nova (2002), as DMUs (Decision Making Units) podem ser grupos empresariais, empresas individuais, unidades administrativas, mas devem atender aos seguintes requisitos: as unidades em análise devem ser comparáveis; devem atuar sob as mesmas condições; os fatores (insumos e produtos) devem ser os mesmos para cada unidade, diferindo apenas na intensidade e magnitude.

O objetivo é construir um conjunto de referências a partir dos dados das DMUs e, então, classificá-las em eficientes ou ineficientes, tendo como referencial essa superfície formada. Assim, uma pressuposição fundamental na técnica DEA é a de que, se uma DMU A é capaz de produzir $\mathrm{Y}(\mathrm{A})$ unidades de produto utilizando $\mathrm{X}(\mathrm{A})$ unidades de insumos, outras DMUs também podem fazê-lo, caso estejam operando eficientemente. A medida de eficiência é relativa 
e o respectivo valor para uma unidade de produção corresponde ao desvio observado em relação àquelas unidades consideradas eficientes (GOMES; BAPTISTA, 2004).

Assim, para a execução dos modelos DEA, são necessários dados referentes aos insumos e produtos de cada DMU a ser analisada. Charnes et al. (1981) identificam alguns pontos para auxiliar na escolha dos dados, tais como: os dados não devem assumir valores negativos; os insumos, os produtos e a escolha das DMUs devem refletir o interesse do pesquisador; a unidade de medida dos insumos e produtos não necessita ser a mesma; e, preferencialmente, o número de variáveis (soma de insumos e produtos) não deve superar em um terço o número de observações (GOMES; BAPTISTA, 2004).

Para utilização da análise envoltória de dados, algumas pressuposições devem ser aceitas, como: a) as DMUs devem ser compostas pelo mesmo conjunto de insumos e produtos; b) as DMUs devem ser autônomas na tomada de decisão; e c) as DMUs devem ser homogêneas e operar na mesma unidade de medida (FERREIRA, 2005).

\section{VARIÁVEIS DO MODELO}

Tendo com referência os recursos físicos e humanos utilizados nos hospitais, serão escolhidas as seguintes variáveis para compor a análise da eficiência.

\section{Recursos/ Insumos - inputs}

Nessa categoria incluem-se os inputs representativos dos esforços que os diversos tipos de mão de obra fazem para atingir os objetivos dos hospitais. Incluem-se também os inputs de capital relacionados à capacidade física e operacional dos hospitais.

-Equipamentos existentes/em uso: representados por equipamentos de diagnósticos por imagens;

- Profissionais: como médicos, enfermeiros e colaboradores;

- Estrutura: número de leitos, capacidade de atendimento diário.

\section{Produtos/ Serviços - outputs}

Relacionado aos outputs, será analisado o processo pelo qual o paciente passou na unidade hospitalar.

- Produção ambulatorial (representada por serviços ambulatoriais);

- Número de pacientes internados;

- Cirurgias realizadas.

Tabela 1 - Resumo das variáveis utilizadas no modelo

\begin{tabular}{cc}
\hline Variáveis & Insumos/Produtos \\
\hline Número de leitos em operação & Inputs \\
Número de Funcionários não médicos & Inputs \\
Número de Médicos & Inputs \\
Número de equipamentos de diagnóstico & Inputs \\
\hline Número de pacientes internados & Outputs \\
Número de pacientes ambulatoriais & Outputs \\
Número de Cirurgias & Outputs \\
Número de pacientes atendidos & Outputs \\
\hline
\end{tabular}

Fonte: Dados da própria pesquisa

\section{OS MODELOS UTILIZADOS}

Em virtude da necessidade de atendimento e da alta demanda, pode-se supor que as organizações hospitalares operam sempre (ou deveriam operar) com excesso de capacidade, é 
o que afirmam ver Fare et al. (1989). Partindo desse pressuposto, o equilíbrio de curto prazo não seria frequente em tais organizações. $\mathrm{O}$ modelo $\mathrm{CCR}$ pressupõe a presença de retornos constantes de escalas, cuja existência, de acordo com a teoria microeconômica, é mais usual quando são considerados prazos mais longos (MARINHO, 2001).

Os modelos escolhidos são orientados no sentido dos outputs (output oriented) por questões que podem ser, principalmente, identificadas na natureza de serviços dos hospitais sob análise. Os quantitativos de alguns de seus principais insumos não podem ser reduzidos, pelo menos no sistema tomado como um todo. Médicos e demais funcionários (razoavelmente) estáveis e instalações não podem ser vendidas. Aceita tal hipótese, a pressuposição de maximização de outputs, determinados os inputs.

\section{RESULTADOS E DISCUSSÃO}

Na Tabela 1 são apresentadas as variáveis empregadas no modelo de eficiência, com suas estatísticas descritivas.

Destaca-se na Tabela 2 a participação de hospitais de diferentes portes, o que resultou em hospitais com número de leitos que variam de 40 a 1.164. Considerando a média de aproximadamente 278 leitos, destaca-se a ampla abrangência da amostra que contempla, em um só escopo, pequenas, médias e grandes instituições hospitalares.

Tabela 2 - Estatística descritiva das variáveis

\begin{tabular}{|c|c|c|c|c|c|}
\hline Variáveis & $\mathbf{n}$ & Mínimo & Máximo & Média & Desvio-padrão \\
\hline \multicolumn{6}{|l|}{ Inputs } \\
\hline Leitos & 35 & 40 & 1164 & 278,06 & 216,76 \\
\hline Médicos & 35 & 9 & 7332 & 1444,29 & 2008,32 \\
\hline Colaboradores não médicos & 35 & 47 & 13045 & 1827,37 & 2721,72 \\
\hline Número de equipamentos diagnósticos & 35 & 0 & 205 & 19,06 & 33,69 \\
\hline \multicolumn{6}{|l|}{ Outputs } \\
\hline $\mathrm{n}^{\circ}$ de internações & 35 & 625 & 48881 & 12244,94 & 9790,03 \\
\hline $\mathrm{n}^{\circ}$ de atendimento ambulatorial & 35 & 0 & 1583127 & 198253,14 & 305295,20 \\
\hline Número de cirurgias & 35 & 0 & 37866 & 8438,06 & 8784,84 \\
\hline
\end{tabular}

Fonte: Dados da pesquisa.

Dentre as variáveis consideradas inputs, a que apresentou maior desvio-padrão, ou seja, a variável com maior discrepância entre o valor mínimo e máximo, conforme a Tabela 2, foi a variável "colaboradores não médicos". Esta constatação indica uma grande diferenciação na capacidade operacional dos hospitais estudados. Conforme a Tabela 2, a variável com maior desvio-padrão, dentre aquelas variáveis consideradas outputs, é a variável "total de procedimentos ambulatoriais que podem estar relacionadas às diferentes capacidades ambulatoriais". Desse modo, considera-se que os dados estão bem dispersos e são válidos para a presente análise.

A Tabela 3 apresenta a análise de eficiência dos hospitais, realizada através do cálculo do DEA. O primeiro resultado a ser assinalado a partir da aplicação de DEA é o ranking dos hospitais, que se reproduz na Tabela 3 , em que os escores dos 35 hospitais foram obtidos com o modelo CCR e com dados para 2012. 
Tabela 3 - Escore de Eficiência dos Hospitais Estudados

\begin{tabular}{|c|c|c|}
\hline HOSPITAIS & ESCORE & TIPO \\
\hline HOSPITAL JOAO XXIII & $100,00 \%$ & PUBLICO \\
\hline HOSPITAL DAS CLINICAS DA UFMG & $100,00 \%$ & PUBLICO \\
\hline HOSPITAL INFANTIL JOAO PAULO II & $100,00 \%$ & PUBLICO \\
\hline HOSPITAL ALBERTO CAVALCANTI & $100,00 \%$ & PUBLICO \\
\hline HOSPITAL GALBA VELLOSO & $100,00 \%$ & PUBLICO \\
\hline HOSPITAL E MATERNIDADE THEREZINHA DE JESUS & $100,00 \%$ & FUNDAÇÃO \\
\hline HOSPITAL FELICIO ROCHO & $100,00 \%$ & FUNDAÇÃO \\
\hline HOSPITAL ISRAELITA ALBERT EINSTIN & $100,00 \%$ & FUNDAÇÃO \\
\hline HOSP. PAULO DE TARSO & $100,00 \%$ & PUBLICO \\
\hline HOSPITAL LIFECENTER & $100,00 \%$ & PRIVADO \\
\hline HOSP. MATER DEI & $100,00 \%$ & PRIVADO \\
\hline HOSPITAL MERIDIONAL & $100,00 \%$ & PRIVADO \\
\hline HOSPITAL OSWALDO CRUZ & $85,30 \%$ & FUNDAÇÃO \\
\hline HOSPITAL REGIONAL ANTONIO DIAS & $84,51 \%$ & PUBLICO \\
\hline HOSPITAL SAO SEBASTIAO & $82,91 \%$ & FUNDAÇÃO \\
\hline HOSPITAL DA BALEIA & $71,12 \%$ & FUNDAÇÃO \\
\hline HOSPITAL SAMARITANO & $68,33 \%$ & FUNDAÇÃO \\
\hline HOSP. NOSSA SENHORA DAS DORES & $67,64 \%$ & FUNDAÇÃO \\
\hline HOSPITAL JULIA KUBITSCHEK & $65,53 \%$ & PUBLICO \\
\hline HOSPITAL SIRIO LIBANES & $62,59 \%$ & FUNDAÇÃO \\
\hline HOSPITAL NIPO BRASILEIRO & $59,52 \%$ & FUNDAÇÃO \\
\hline HOSPITAL METROPOLITANO S A & $59,12 \%$ & PRIVADO \\
\hline HOSPITAL SANTA CATARINA & $58,84 \%$ & FUNDAÇÃO \\
\hline HOSPITAL 9 DE JULHO AS & $58,54 \%$ & PRIVADO \\
\hline HOSP. ARNALDO GAVAZZA & $56,27 \%$ & FUNDAÇÃO \\
\hline HOSPITAL CESAR LEITE & $54,92 \%$ & FUNDAÇÃO \\
\hline IRMANDADE DO HOSP. POÇOS DE CALDAS & $52,84 \%$ & FUNDAÇÃO \\
\hline HOSPITAL SÃO CAMILO POMPEIA & $49,93 \%$ & FUNDAÇÃO \\
\hline HOSPITAL BANDEIRANTES & $46,74 \%$ & FUNDAÇÃO \\
\hline HOSPITAL SÃO SEBASTIAO DE RAUL SOARES & $46,74 \%$ & FUNDAÇÃO \\
\hline HOSPITAL REGIONAL JOAO PENIDO & $45,35 \%$ & PUBLICO \\
\hline HOSPITAL QUINTA DOR & $36,04 \%$ & PRIVADO \\
\hline SANTA CASA DE MISERICORDIA DE BH & $34,55 \%$ & FUNDAÇÃO \\
\hline HOSPITAL NSH DA CONCEIÇÃO DE RIO CASCA & $34,26 \%$ & FUNDAÇÃO \\
\hline HOSPITAL GERAL DE BARBACENA DR JOSE AMERICO & $25,46 \%$ & PUBLICO \\
\hline
\end{tabular}

Fonte: Dados da pesquisa.

Pode ser observado, através dos resultados, que apenas 12 dos 35 hospitais estudados podem ser considerados eficientes. Isto demonstra a baixa capacidade destas instituições para gerar resultados (produtos). Assim, percebe-se que alguns hospitais conseguem, com menor alocação de recursos, gerar melhores resultados. 
Outra análise interessante é a relação entre o tamanho dos hospitais e a eficiência. Segundo Marinho e Façanha (2001), vários trabalhos já fizeram essa análise, como Byrnes e Valdmanis (1994), que sugeriram que a curva de custos dos hospitais segue "(...) a curva de custos tradicional com formato de U", ou seja, que os hospitais de tamanho "médio" (no caso, de aproximadamente 230 leitos) tendem a ser mais eficientes. Já Ferrier e Valdmanis (1996) afirmam que "(...) grandes e pequenos hospitais são relativamente mais eficientes tecnicamente do que os hospitais de tamanho médio". Eakin (1991), por sua vez, afirma que "(...) o tamanho do hospital, medido pelo número de leitos, é positivamente correlacionado com ineficiências alocativas". Já Marinho e Façanha (2001) concluíram que a média da eficiência técnica dos hospitais que possuem até 200 leitos é 32,73\% maior do que a média da eficiência dos hospitais que oferecem mais do que 200 leitos.

$\mathrm{Na}$ Tabela 4 podemos observar os valores encontrados nesta pesquisa:

Tabela 4 - Número de Leitos versus Eficiência Técnica dos Hospitais

\begin{tabular}{|c|c|c|r|c|}
\hline Número de Leitos & \multicolumn{1}{l|}{ N } & Média de Eficiência & Desvio Padrão & \multicolumn{1}{|c|}{ Sign. T } \\
\hline Até 200 & 19 & $69,98 \%$ & $24,24933 \%$ & \multirow{2}{*}{0,473} \\
\hline Acima de 200 & 16 & $67,90 \%$ & $22,75811 \%$ & \\
\hline
\end{tabular}

Fonte: Dados da pesquisa.

A eficiência, assim como no artigo de Marinho e Façanha (2001), é, aparentemente, função decrescente nos hospitais maiores. Entretanto, é necessário avaliar a validade estatística da diferença de médias entre a eficiência dos pequenos e grandes hospitais. Através da aplicação do teste t para amostras independentes, observa-se que a significância estatística é de 47,3\%, bem acima do limite de aceitação de $5 \%$, demonstrando que estatisticamente o porte do hospital não influencia sua eficiência, contrapondo os trabalhos citados acima.

Outro ponto relevante a ser pesquisado é sobre quais tipos de instituições são mais eficientes. Assim, a Tabela 5 apresenta a média de eficiência dos 3 tipos estudados nesta pesquisa.

Tabela 5 - Escores de eficiência técnica dos hospitais

\begin{tabular}{l|c|c|c|c|c}
\hline Tipos de Hospitais & N & Unidades eficientes (\%) & Média & Mínimo & Máximo \\
\hline Públicos & 09 & 55,56 & $80,09 \%$ & $25,46 \%$ & $100 \%$ \\
\hline Filantrópicos & 19 & 15,79 & $64,87 \%$ & $34,26 \%$ & $100 \%$ \\
\hline Privados & 07 & 57,14 & $75,62 \%$ & $36,04 \%$ & $100 \%$ \\
\hline
\end{tabular}

Fonte: Dados da pesquisa.

Observa-se que os hospitais públicos possuem maior média de eficiência que os demais tipos de hospitais. Os hospitais filantrópicos obtiveram o menor desempenho em relação à média e também no percentual de unidades eficientes, sendo considerado eficiente apenas um $(15,79 \%)$ dos 19 hospitais estudados.

Contudo, é necessário observar se estas diferenças são estatisticamente significantes, conforme pode ser observado na Tabela 6 .

Tabela 6 - Teste t para comparação de médias de eficiência entre os hospitais

\begin{tabular}{|c|c|}
\hline Comparação & Significância T \\
\hline Público - Privado & 0,098 \\
\hline Público - Filantrópico & 0,032 \\
\hline Privado - Filantrópico & 0,047 \\
\hline
\end{tabular}

Fonte: Dados da pesquisa. 
Após a aplicação do teste $t$ para amostras independentes, os resultados demonstram que os hospitais filantrópicos podem ser considerados mais ineficientes que os demais tipos, já que as duas comparações de médias foram estatisticamente significantes a 5\%. Já em relação a comparação entre os hospitais públicos e privados, só se pode afirmar que há diferença de médias se for aceita uma chance de erro de $10 \%$. Contudo, é necessário ressaltar que a limitação da amostra contribuiu para a alta significância.

Os resultados contrapõem a hipótese inicial da pesquisa, que esperava maior ineficiência dos hospitais públicos, devido à histórica cultura de ineficiência da gestão pública brasileira e pela maior necessidade de procedimentos formais e normativos. Contudo, estes hospitais demonstraram maior nível de eficiência.

Além dessas análises, buscou-se avaliar a relação entre a eficiência dos hospitais estudados, que está ligada às suas atividades operacionais, e o desempenho financeiro desses hospitais. O primeiro passo foi levantar o lucro dos hospitais privados e apurar o superávit/déficit dos públicos e aplicar um teste $\mathrm{t}$ para comparação de médias de grupos independentes, objetivando avaliar se os hospitais eficientes apresentam melhor resultado líquido. Os resultados podem ser vistos na Tabela 7 abaixo:

Tabela 7 - Resultado Financeiro versus Eficiência Técnica dos Hospitais

\begin{tabular}{|c|c|c|c|c|}
\hline Número de Leitos & N & Resultado Médio & Desvio Padrão & Sign. T \\
\hline Ineficientes & 23 & $30.268,19$ & $60.859,48$ & \multirow{2}{*}{0,021} \\
\hline Eficientes & 12 & $14.739,98$ & $12.698,97$ & \\
\hline
\end{tabular}

Fonte: Dados da pesquisa.

É importante ressaltar que o resultado líquido foi dividido pelo número de leitos, para trazer todos os hospitais para o mesmo horizonte de comparação e os resultados não serem afetados pela diferença de porte entre eles.

Observa-se que os hospitais ineficientes apresentaram maior média no resultado financeiro que os eficientes, o que demonstra uma relação negativa entre eficiência técnica e resultado financeiro.

Estes resultados podem ser melhores entendidos pela contratualização que o SUS tem com os hospitais públicos e filantrópicos. Os hospitais têm um pacto de quantidade de atendimentos que devem ser realizados e, caso ultrapassem o número de atendimentos contratualizados, estes não irão gerar fluxos de caixa imediatos. Sendo assim, existe uma tendência de que os hospitais eficientes atendam mais que o contratualizado, já que eles apresentam melhores produtos. Em contrapartida, estes melhores resultados geram maiores custos variáveis e, como eles não são reembolsados imediatamente, os hospitais terão sua margem de contribuição afetada e, consequentemente, seus lucros.

Isto demonstra uma necessidade de revisão dos métodos e quantidade de recursos que o SUS repassa para os hospitais, para estimular maior eficiência na geração de produtos (atendimentos), que deve ser o objetivo principal da saúde pública brasileira.

Além do resultado financeiro, foi analisada a relação entre a eficiência e alguns índices financeiros da empresa. Ressalta-se que, nestas análises, não foi possível incluir os hospitais públicos, já que eles não apresentam informações suficientes para o cálculo dos índices. Os resultados podem ser observados na Tabela 8 . 
Tabela 8: Resultado da média dos indicadores financeiros

\begin{tabular}{ccccc}
\hline Índice & Eficiência & $\mathbf{N}$ & Média & Desvio Padrão \\
\hline \multirow{2}{*}{ LIQGERAL } & Ineficientes & 19 & 1,0879 &, 92069 \\
& Eficientes & 7 & 1,4543 & 1,55772 \\
\hline \multirow{2}{*}{ LIQCORRENTE } & Ineficientes & 19 & 1,57905 & 1,087301 \\
& Eficientes & 7 & 2,51429 & 1,531621 \\
\hline \multirow{2}{*}{ LIQSECA } & Ineficientes & 19 & 1,45905 & 1,060265 \\
& Eficientes & 7 & 2,33571 & 1,622476 \\
\hline \multirow{2}{*}{ MARGLIQUIDA } & Ineficientes & 19 & $8,9674 \%$ & $35,73714 \%$ \\
& Eficientes & 7 & $6,0186 \%$ & $14,59753 \%$ \\
\hline \multirow{2}{*}{ RENTDOATIVO } & Ineficientes & 19 & $1,1126 \%$ & $10,98074 \%$ \\
& Eficientes & 7 & $1,7300 \%$ & $16,25239 \%$
\end{tabular}

Fonte: Dados da Pesquisa

Destaca-se que, ao comparar o índice de liquidez geral, a média dos eficientes é maior que a dos ineficientes, sendo ambos maiores que 1. Partindo do pressuposto de que quando se trata de liquidez o indicador é "quanto maior melhor", as entidades em média apresentam um resultado satisfatório, uma vez que a liquidez geral leva em consideração a situação de curto e longo prazo. Como destacado, os índices de liquidez corrente e liquidez seca são maiores que 1, a liquidez corrente refletindo a capacidade de pagamento da entidade em curto prazo, uma vez que o crescimento exagerado das contas a receber, principalmente, deve ser analisado, pois poderá ocasionar um aumento de inadimplência. Contudo o estoque dos hospitais não tem comprometido sua liquidez, uma vez que o giro do estoque é diário, até mesmo para suprir a demanda e eficiência no seu controle. Não se estoca mais que o necessário.

Os resultados demonstram uma relação positiva entre a liquidez e a eficiência, sugerindo uma melhor capacidade de cumprir com os compromissos financeiros.

Com relação aos índices de rentabilidade, observa-se uma relação oposta, já que é demonstrado pela análise da lucratividade que os hospitais eficientes têm menor capacidade de geração de resultados financeiros. Fato facilmente explicável, uma vez que os ineficientes não possuem controles eficientes de sua receita, pois na maioria das vezes o seu faturamento é bem superior a sua capacidade de atendimento.

\section{LIMITAÇÕES DA PESQUISA}

Salientam La Forgia e Couttolenc (2009), assim como ocorre com a maioria das pesquisas em relação a este assunto, que os estudos brasileiros sobre eficiência hospitalar apresentam algumas "limitações". Primeiramente, as pesquisas de modo geral se utilizam de amostras pequenas.

Em relação à amostra, é preciso destacar a restrição de acesso aos dados. Especificamente em relação ao cálculo dos indicadores operacionais, foram utilizados os dados coletados no DATASUS e no CNES. Por meio de acesso a base de dados, é possível obter informações, tais como números de leitos em uso, profissionais assistenciais vinculados à entidade, números de procedimentos e atendimentos realizados. Vale ressaltar que, para a 
presente pesquisa, foram utilizados apenas dados das tabelas Tbwin-Data, considerados os mais relevantes para análise.

Dificilmente é possível obter informações operacionais dos hospitais brasileiros. Isso porque as entidades, de certa forma, consideram tais dados como confidenciais e normalmente não divulgam para o público. Diante do mencionado supra, optou-se por considerar os dados disponíveis no CNES e DATASUS.

Outra restrição é o acesso aos dados para o cálculo dos indicadores financeiros. Para o presente trabalho foram coletadas informações de organizações hospitalares que publicassem suas demonstrações financeiras para o período determinado para análise. Neste caso, ficou impossibilitado o cálculo para os hospitais públicos.

\section{CONCLUSÃO}

Como mencionado no decorrer deste trabalho, os hospitais são reconhecidos como organizações complexas. A combinação eficaz dos recursos humanos, tecnológicos e financeiros necessários a essas organizações exige dos gestores ideias inovadoras, tais como implementação do sistema de custos, estabelecer convênios que agregam receitas e uma experiência profissional para garantir uma gerência eficaz e eficiente.

A proposta de uma estrutura de avaliação da eficiência alocativa de hospitais baseou-se nos diferentes estudos sobre a DEA realizados em hospitais. Contudo, DEA é considerada pelos autores uma importante ferramenta de apoio a decisões no sistema de Saúde.

Com base no modelo de programação Linear - DEA, o objetivo geral foi analisar se existe diferença na eficiência alocativa dos recursos econômico-financeiros dos hospitais públicos, filantrópicos e privados.

O modelo demonstrado no presente trabalho agrupou 35 hospitais com base nos indicadores operacionais, inputs e outputs. A utilização de tais indicadores possibilitou a comparabilidade da eficiência entre os hospitais. Nesse sentido, os hospitais foram segregados em três grupos: públicos, filantrópicos e privados.

Com relação aos resultados alcançados, verificou-se que entre os mais eficientes dentro da amostra de 35 hospitais estão os públicos e os privados, ficando os filantrópicos com menor número de hospitais eficientes, uma vez que foram analisados um número maior de hospitais filantrópicos.

Tal fato pode ser explicado porque os hospitais públicos estudados fazem parte de uma fundação pública que administra diretamente os recursos operacionais e financeiros. Outro ponto importante é que os hospitais públicos filiados à FHEMIG apresentam um sistema de custos implantado, que os diferencia muitas vezes dos demais hospitais. Durante a pesquisa foi possível observar que os hospitais públicos estudados não atendem mais que sua capacidade produtiva, confirmando assim os resultados obtidos no presente trabalho.

Grande parte dos hospitais filantrópicos estudados não apresentam em sua estrutura organizacional um sistema de custos implementado. Com base nos dados da pesquisa, tais hospitais estão sempre atendendo além da capacidade, muitas vezes para garantir o título de filantropia.

Após avaliar a relação da eficiência com os indicadores financeiros, pontua-se que, quando se trata de liquidez, existe uma relação positiva com a eficiência, ou seja, os hospitais eficientes apresentam uma média superior de liquidez que os hospitais ineficientes.

De modo geral, conclui-se que o presente trabalho pode contribuir para a análise do desempenho da gestão financeira e produtiva dos hospitais. A priori, porque apresenta a relação de indicadores financeiros e operacionais que podem ser utilizados para análise da eficiência dessas entidades. 
Acredita-se, em particular, que o estudo aqui realizado pode ser útil para fins de orientação aos gestores de organizações hospitalares, como administrar os resultados obtidos com a capacidade produtiva.

Para pesquisas futuras, espera-se ampliar a amostra de hospitais analisados, quem sabe por regiões geográficas, a fim de confirmar ou contrapor os resultados encontrados. Coletar dados de diferentes anos, para que seja possível comparar a eficiência alocativa dos hospitais ao longo do tempo.

\section{CONCLUSÃO}

Como mencionado no decorrer deste trabalho, os hospitais são reconhecidos como organizações complexas. A combinação eficaz dos recursos humanos, tecnológicos e financeiros necessários a essas organizações exigem dos gestores ideias inovadoras, tais como implementação do sistema de custos, estabelecer convênios que agregam receitas e uma experiência profissional para garantir uma gerência eficaz e eficiente.

A proposta de uma estrutura de avaliação da eficiência alocativa de hospitais baseou-se nos diferentes estudos sobre a DEA realizados em hospitais. Contudo, DEA é considerada pelos autores uma importante ferramenta de apoio à decisões no sistema de Saúde.

Baseado no modelo de programação Linear-DEA, o objetivo geral foi analisar se existe diferença na eficiência alocativa dos recursos econômicos financeiros dos hospitais públicos, filantrópicos e privados.

O modelo demonstrado no presente trabalho agrupou 35 hospitais com base nos indicadores operacionais, inputs e outputs. A utilização de tais indicadores possibilitou a comparabilidade da eficiência entre os hospitais. Nesse sentido, os hospitais foram segregados em três grupos, os públicos, filantrópicos e privados.

Com relação aos resultados alcançados, verificou-se que entre os mais eficientes dentro da amostra de 35 hospitais estão os públicos e os privados, ficando os filantrópicos com menor número de hospitais eficientes, uma vez que foram analisados um número maior de hospitais filantrópicos.

Tal fato pode ser explicado, pois, os hospitais públicos estudados fazem parte de uma fundação pública que administram diretamente os recursos operacionais e financeiros. Outro ponto importante que os hospitais públicos filiados a FHEMIG apresentam um sistema de custos implantado, que diferencia muitas vezes dos demais hospitais. Durante a pesquisa foi possível observar que os hospitais públicos estudados não atendem mais que sua capacidade produtiva, confirmando assim os resultados obtidos no presente trabalho.

Grande parte dos hospitais filantrópicos estudados não apresentam em sua estrutura organizacional um sistema de custos implementado. Com base nos dados da pesquisa tais hospitais estão sempre atendendo além da capacidade, muitas das vezes para garantir o titulo de filantropia.

Após avaliar a relação da eficiência com os indicadores financeiros, pontua-se que quando se trata de liquidez existe uma relação positiva com a eficiência, ou seja, os hospitais eficientes apresentam uma média superior de liquidez que os hospitais ineficientes.

De modo geral, conclui-se que o presente trabalho pode contribuir para analise do desempenho da gestão financeira e produtiva dos hospitais. A priori, porque apresenta a relação de indicadores financeiros e operacionais que podem ser utilizados para analise da eficiência dessas entidades.

Acredita-se, em particular, que o estudo aqui realizado pode ser útil para fins de orientação aos gestores de organizações hospitalares, como administrar os resultados obtidos com a capacidade produtiva. 
Para pesquisas futuras, espera-se ampliar a amostra de hospitais analisados, quem sabe por regiões geográficas a fim de confirmar ou contrapor os resultados encontrados. Coletar dados de diferentes anos, para que seja possível comparar a eficiência alocotiva dos hospitais ao longo dos anos.

\section{REFERÊNCIAS BIBLIOGRÁFICAS}

ATHAYDE, A.H. Avaliação de desempenho do transporte rodoviário de cargas pelo método de análise envoltória de dados - DEA. 2003. São Carlos. Dissertação (Mestrado em Engenharia dos Transportes). Escola de Engenharia de São Carlos, Universidade de São Paulo.

BANKER, R.D. et.al. Some models for estimating technical and scale inefficiencies in data envelopment analysis. Management Science, v. 30, p. 1078- 1092, 1984.

BITTAR.O.J.N.V. Indicadores de Qualidade e quantidade em saúde. RAS -Vol. 3, № 12 - Jul-Set, 2001.

BRAGA, J. C. S. \& GOES DE PAUlA, 1981. Saúde e Previdência - Estudos de Política Social. São Paulo:Cebes-Hucitec.

BRASIL. Decreto no ${ }^{\mathbf{7 6 . 9 7 3}}$, de 31 de dezembro de 1975.

BRASIL. Ministério da Saúde. DATASUS. Disponível em: <http://www2.datasus.gov.br/DATASUS/index.php?area=04 >. Acesso em: 20 de agosto 2013.

BYRNES, P., VALDMANIS, V. Analyzing technical and allocative efficiency of hospitals. In: CHARNES, A., COOPER, W. W., LEWIN, A. Y., SEIFORD, L. M. (eds.). Data Envelopment Analysis. London: Kluwer Academic Publishers, p. 129-144, 1994.

CALVO, M.C. Hospitais públicos e privados no Sistema Único de Saúde do Brasil: o mito da eficiência privada no Estado de Mato Grosso em 1998. 2002.223f. Tese ( Doutorado em Engenharia da Produção) - Universidade Federal de Santa Catarina, Florianópolis, 2002.

CASA NOVA, S.P.C. Utilização da análise por envoltória de dados (DEA) na análise de demonstrações contábeis. Tese de doutorado da Universidade de são Paulo, São Paulo, 2002.

CELESTINO, P. Nó de Normas. Notícias Hospitalares. Gestão de Saúde em Debate, vol. 4, n. 39, out./nov. 2002.

CESCONETTO, A. et al. Avaliação da eficiência produtiva de hospitais do SUS de Santa Catarina, Brasil. Cadernos de Saúde Pública, Rio de Janeiro, 24(10):2407-2417, out, 2008.

CHARNES, A.; COOPER, W. W.; RHODES, E. Evaluating program a managerial efficiency: an application of Data Envelopment Analysis to program follow through. Management Science, v. 27, n. 6. p. 668-697, 1981.

CHARNES, A.et al. Measuring the efficiency of decision-making units. European Journal of Operational Research, v. 2, p. 429-444, 1978.

CNES - Cadastro Nacional de Estabelecimento de Saúde. Disponível em; <http://cnes.datasus.gov.br/>. Acesso em 10 de julho de 2013.

COSTA, F. L.; CASTANHAR, J. C. Avaliação de programas públicos: desafios conceituais e metodológicos. Revista de Administração Pública, v. 37, n. 5, p. 969-92, set./out. 2003.

EAKIN, B. K. Allocative inefficiency in the production of hospital services. Southern Economic Journal, v. 58, n. 1, p. 240-248, July 1991. 
FARE, R.et al. Capacity, competition and efficiency in hospitals: a nonparametric approach. The Journal of Productivity Analysis, n. 1, p. 123-138, 1989

FARREL, M.J. The measurement of productive efficiency. Journal of the Royal Statistic Society. p. 253-290, 1957.

FEDERAÇÃO BRASILEIRA DE HOSPITAIS. Indicadores de saúde. Brasília 2012. Disponível em <http://fbh.com.br/category/institucional/publicacoes/indicadores-de-saude/>. Acessado em 20 de abr. 2013.

FERREIRA, M. A. M. Eficiência técnica e de escala de cooperativas e sociedades de capital na indústria de laticínios do Brasil. Tese (Doutorado em Economia Aplicada) - Universidade Federal de Viçosa, Viçosa, 2005.

FERRIER, G. D., VALDMANIS, V. Rural hospital performance and its correlates. The Journal of Productivity Analysis, v. 7, p. 63-80, 1996.

FONSECA, P. C.; FERREIRA, M. A. M. Investigação dos Níveis de Eficiência na utilização de Recursos no Setor de Saúde: uma análise das microrregiões de Minas Gerais. Saúde e Sociedade. São Paulo, v.18, n.2, p.199-213, 2009.

GIL, A. C. Como Elaborar Projeto de Pesquisa. 5 ed. São Paulo: Atlas, 2010.

GOMES, A. P.; BAPTISTA, A. J. M. S. Análise envoltória de dados: conceitos e modelo básicos. In: SANTOS, M. L. dos; VIEIRA, W. da C. (Orgs.). Métodos quantitativos em Economia. Viçosa: Editora UFV, 2004.

GONÇALVES, A. C. et al. Análise Envoltória de Dados na avaliação de Hospitais públicos nas capitais brasileiras. Saúde pública 2007.

LA FORGIA, G. M.; COUTTOLENC, B. F. Desempenho hospitalar brasileiro: em busca da excelência. São Paulo: Singular, 2009.

LIMA, S. M. L. et al. Caractlerização gerencial dos hospitais filantrópicos no Brasil. Caderno de Saúde Pública, Rio de Janeiro, v. 20, n. 5, p.1249-1261, set.-out. 2004.

LINS, M. P. E.; et al. O uso da Análise Envoltória de Dados (DEA) para avaliação de hospitais universitários brasileiros. Ciência \& Saúde Coletiva, 12(4):985-998, 2007.

LOBO E LINS, M. P. E. (2011) Avaliação da eficiência dos serviços de saúde por meio da Análise Envoltória de Dados, Caderno de Saúde Coletiva, Rio de Janeiro.

LOBO.M.S.C. Aplicação da análise envoltória de dados (DEA) para apoio às políticas públicas de saúde: o caso dos hospitais de ensino. 2010. Tese ( Doutorado em Engenharia de Produção)- Universidade Federal do Rio de Janeiro, Rio de Janeiro/RJ,2010.

MARINHO, A. \& FAÇANHA, L. O. (2001a) Hospitais Universitários: Avaliação comparativa de eficiência técnica. Texto para discussão $\mathrm{N}^{\circ} 805$. Rio de Janeiro, RJ: IPEA.

MARINHO, A. Estudo de eficiência em alguns hospitais públicos e privados com a geração de rankings. Rio de Janeiro: IPEA, 2001.

MARRACINI. P. Administração estratégica hospitalar: Um estudo de caso de hospital privado filantrópico inserido no sistema único de saúde. 2002. Dissertação (Mestrado em Administração de Empresas)- FECAP, São Paulo.

MILLER, R. L. Microeconomia: teoria, questões e aplicações. Tradução Sara Gedanke. São Paulo: McGrawHill do Brasil, 1981.

OMS-ORGANIZAÇÃO MUNDIAL DE SAUDE. Indicadores de Saúde 2011. Disponível em<http://new.paho.org/bra/index.php?option=com_content \&view=category\&layout=blog\&id=758\&Item

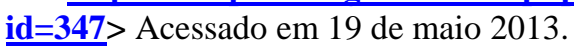


PINDYCK, R. S.; RUBINFELD, D. L. Microeconomia: teoria microeconômica. São Paulo: Makron Books, 1994.

PORTELA M. C . et al. Caracterização assistencial de hospitais filantrópicos no Brasil .rev. Saúde publica 2004;38(6): 811-8.

RAIMUNDINI, S. L. et al. Aplicabilidade do sistema ABC e análise de custos hospitalares: comparação entre hospital público e hospital privado. XXVIII Encontro da ANPAD. Anais... Curitiba, 2004.

RAIMUNDINI,S.L. Aplicabilidade do sistema ABC e análise de custos: estudo de caso em hospitais públicos. 2003. Dissertação (Mestrado em Administração) - Departamento de Administração, Universidade Estadual de Maringá, Maringá/PR, 2003.

ROTTA, C. Utilização de indicadores de desempenho hospitalar como instrumento gerencial. $2004.124 \mathrm{f}$. Tese ( Doutorado em Saúde Pública administração hospitalar) - Faculdade de Saúde Pública, Universidade de São Paulo, 2004.

SEDIYAMA,N.Y.M. et al. Análise da eficiência de hospitais filantrópicos de pequeno porte pela Análise Envoltória de Dados (DEA). Encontro de Administração Pública e Governo- ENAPG, 18 a 20 de nov, 2012 Salvador. Anais... Salvador: ANPAD, 2012.

SOUZA, A. A. et al.Controle de Gestão em Organizações Hospitalares. In: XII Congresso de Contabilidade e Auditoria, 2008, Aveiro, Portugal. Anais... 2008.

SOUZA. A.A. et al. Uma análise financeira do hospital metropolitano de urgência e emergência. IX Convibra Administração- Congresso virtual brasileiro de administração, 2012.

WOLFF,L.D.G. Um modelo para avaliar o impacto do ambiente operacional na produtividade dos hospitais brasileiros. 2005. Tese (Doutorado em Engenharia de Produção) - Universidade Federal de Santa Catarina, Florianópolis/SC, 2005. 